\title{
Hybrid exponential-hyperbolic discounting and growth without commitment
}

\author{
Christopher Tsoukis
}

Keele University*

Frédéric Tournemaine

Chulalongkorn University**

Max Gillman

University of Missouri-St. Louis****

\section{March 2017}

\begin{abstract}
We analyse the time-consistent intertemporal behaviour of an individual who discounts the future hyperbolically (HD) in the absence of commitment. In continuous-time, we extend Barro's (1999) analysis of a 'sophisticated' present-bias in a deterministic setup and characterise consumption in an analytically tractable way. Furthermore, we embed this analysis into a 'flexible AK' model. Greater present bias increases the consumption-capital ratio, decreases the steady-state growth rate, while it increases the interest rate-growth rate wedge. Dynamically, as the interest rate fluctuates over the business cycle, a greater present bias causes the consumption-output ratio to be more procyclical and volatile, thus helping to resolve the 'consumption-output puzzle'. In the transitional dynamics, greater present bias causes a lower steady state capital stock. All these effects are only present or at least more pronounced under HD than present value-equivalent exponential discounting.
\end{abstract}

Keywords:

JEL classification number:
Hyperbolic discounting, growth, business cycles, time inconsistency D910, E13, E210, O410

*Address: Economics and Finance, Keele Management School, Keele University, Staffordshire, ST5 5BG, UK; email: c.tsoukis@ keele.ac.uk

**Address: Faculty of Economics, Chulalongkorn University, Phayathai Road, Pathumwan, Bangkok, 10330, Thailand; Frederic.T@ chula.ac.th

***Address: University of Missouri at St Louis, 1 University Boulevard 408 SSB St Louis, Missouri 6312, USA; gillmanm@umsl.edu

\section{Acknowledgments:}

Max Gillman kindly acknowledges the support of Czech Science Foundation GACR 13-34096S.

This paper has been presented at the Association for Public Economic Theory Conference (PET15), Luxembourg, July 2015, the Manchester Centre for Growth and Business Cycle Research (CGBCR) $8^{\text {th }}$ Conference, Manchester, July 2016 and the $48^{\text {th }}$ Money, Macro and Finance Annual Conference (MMF2016), Bath September 2016. Thanks are due to the Editor and three anonymous referees of The Manchester School for several helpful comments. 


\section{Introduction}

Growing experimental evidence from humans and non-humans (Ainslie, 1992; Lowenstein and Prelec, 1992; Angeletos, Laibson, Repetto, Tobacman and Weinberg, 2000; Frederick et al., 2002; DellaVigna and Malmendier, 2006; DellaVigna, 2009; Sprenger, 2015) shows a present-bias and impatience in intertemporal decisionmaking, in sharp contrast to the predictions of exponential/geometric discounting. Such behaviour can be captured by a discount factor that takes the form of a generalised hyperbola, leading to the label 'hyperbolic discounting' (HD). ${ }^{1}$ Present bias and HD have important implications for many areas in dynamic macroeconomics (consumer behaviour, saving, pensions) including the theory of growth. There is a growing interest in the effects on growth-related issues as witnessed by Barro (1999), Strulik (2015) and Cabo, Martín-Herrán and Martínez-García (2015). Our paper relates to this strand of literature; specifically, we analyse the effects of hyperbolic discounting within a continuous-time, representative-agent, deterministic growth model.

As is well known since at least Strotz (1956; see also Frederick et al., 2002; Sprenger, 2015), in general, non-exponential/geometric discounting scheme induces time inconsistency: ${ }^{2}$ The same intertemporal dilemma will receive different resolutions as the planning period gets nearer to it in time. If so, the perturbation arguments that underlie the Euler equation are not valid. To resolve this difficulty, one may assume full commitment to the initial plan via e.g. a saving plan or the purchase of illiquid assets such as housing; the analysis of full commitment has been carried out by Barro (1999) and Tsoukis (2014). In this case, the Euler equation is re-instated (optimisation is carried out once only at the beginning of time). But commitment is often not sought even if there are grounds to believe that it may be beneficial (Laibson, 2015; O'Donoghue and Rabin, 2015). Assuming away commitment, the alternative route is to seek a time-consistent maximisation procedure for the representative agent. In continuous time, this has been developed by Barro (1999), following Pollak (1968).

In line with Barro (1999) and much other literature, we postulate a 'sophisticated' presently-biased individual, namely one who is aware of their present bias and internalises the implications. Another approach to dealing with time-inconsistency is to assume that the individual is 'naïvely presently-biased', whereby the individual does not realise that their plans will be changed the next instant; see e.g. Strulik (2015), Caliendo and Adland (2007) and Farzin and Wendner (2014). The 'sophisticated' case is analytically more cumbersome, as the re-optimised plan tomorrow is taken into account today, but more internally consistent. In reality, individuals may not be either perfectly sophisticated or perfectly naïve (see Frederick et al., 2002). ${ }^{3}$

We propose a hybrid exponential-HD discount factor that is both tractable and plausible; we show that the exponential part is required alongside the HD part. Our

\footnotetext{
${ }^{1}$ There is a strand of literature that assumes declining impatience by modelling the discount factor on consumption (see Frederick et al., 2002); this insight is outside our scope.

${ }^{2}$ An exception is suggested by Drouhin (2009) which considers a multiplicatively separable hyperbolic discount function which in fact supports a time-consistent solution.

${ }^{3}$ Note however that neither Barro (1999) nor Strulik (2015) explicitly use such terminology.
} 
model of HD also generalises the 'beta-delta' discounting scheme typically employed in discrete time models (e.g. Laibson, 1997; Harris and Laibson, 2001; Krusell and Smith, 2003). ${ }^{4}$ Laibson (1997) takes credit for re-awakening interest on present bias; he applies his discrete-time setup to a neoclassical model with exogenous growth. Moreover, we make the following advances over other closely related contributions: We considerably extend the analysis of Barro (1999) by fully analysing the case of iso-elastic preferences and endogenous growth, on which Barro (1999) is rather tentative. In extending these results, Cabo, Martín-Herrán and Martínez-García (2015) considers a more general HD discount factor with the same properties in the context of an AK model, much as we do. Our advances over their analysis are several: firstly, we develop explicit dynamics of the composite discount rate and relate that to the interest rate/productivity that follows exogenous dynamics. Secondly, as explained below, we start from a formulation that allows a clear economic interpretation of the composite discount rate (as the consumption-intertemporal resources ratio, the main 'grand ratio' of our balanced growth path), in contrast with their analysis in which such an interpretation is not possible. Finally, Cabo et al. (2015) do not offer closedform results, as we do. In all, within our continuous-time setup, we offer the most integrated and analytically tractable model of HD in growth.

More specifically, the first part of the paper develops a time-consistent maximisation procedure proposed by Barro (1999) under a sophisticated bias and derives and fully characterises the dynamic behaviour of the consumption-intertemporal resources ratio. We then embed this analysis into a continuous-time, flexible AK model and derive closed-form solutions. In terms of steady state, we derive a rich set of results on growth, the consumption-capital ratio, saving and the ' $r$-g' relation (to be explained below). Though the setup involves a representative agent, there are indirect implications for income distribution via this differential. Though the framework is non-stochastic, we allow for a business cycle by letting the exogenous driver fluctuate. Finally, the model provides the foundation towards an extension to flexible labour and a more thorough integration of HD into growth theory.

To pre-amble, we show that the presence of present-bias increases the individual's propensity to consume out of lifetime wealth and reduces saving and the growth rate in the steady state. Furthermore, the wedge between the real interest rate and the growth rate (r-g) also rises; this wedge in the view of Piketty $(2014,2015)$ may increase income inequality (a point on which we do not dwell further). In terms of transitional dynamics, a standard AK model with exponential discounting can be interpreted as a limiting case of the more general framework presented here. We show that HD results in intrinsic dynamics richer than the entirely extraneous dynamics of the standard AK model when the exogenous driver of productivity, here equal to the interest rate, exhibits its own dynamics. Near the benchmark case of no HD, we find that a greater present-bias and intensity of HD makes the consumption-capital ratio more procyclical and variable. In other words, present-bias induces individuals to consume guided more by current and less by permanent income than otherwise. This potentially resolves the long-standing 'consumption-output puzzle' in standard models by bringing the predicted consumption-output ratio closer to that observed in the data. As a result, the level of capital is lower under HD. In other words, in addition

\footnotetext{
${ }^{4}$ Zou et al. (2014) develops a stochastic discount factor, from which we abstract.
} 
to the steady-state growth rate effects, we also point out level effects as a result of transitional dynamics.

An important aspect of all our results is that the form, and not only the amount of discounting, plays a key role. In order to distinguish between amount and form of discounting, we use a present value-equivalent (PVEE) discounting scheme that is purely exponential but does the same amount of discounting in some sense as our hybrid HD scheme. All effects derived under our scheme markedly differ from those under the 'PVEE' scheme. This includes results on both steady-state and transitional dynamics. Thus, there is no observational equivalence: Economies that differ in their pattern of discounting behave quite differently. We discuss related literature briefly before summarising our own findings in Corollary 7.

The remainder of the paper is organised as follows. In Section 2, we present a simple model with HD and derive a set of results related to the marginal propensity to consume. In Section 3, we characterise the transitional dynamics of this variable. In Section 4, we embed this analysis into a standard AK model and derive steady-state and dynamic results. We conclude in Section 5. A series of Online Appendices (A-E) elaborate on the most technical aspects of our results.

\section{Hyperbolic discounting without commitment}

\section{1: Exponential versus hyperbolic discounting (HD)}

As in Barro (1999) and others, the framework assumes an infinitely-lived, representative-agent model where preferences are characterised by HD and there is no commitment mechanism; time is continuous; utility is assumed iso-elastic. At the time of planning $(\mathrm{t})$, the individual is assumed to maximise:

$$
U_{t} \equiv \int_{t}^{\infty} \frac{C_{\tau}^{1-\sigma}}{1-\sigma} \Phi(\tau, t) d \tau
$$

where $\mathrm{C}$ is real consumption and $\sigma$ the inverse of the elasticity of substitution. Empirical evidence suggests that the intertemporal elasticity of substitution is less than unity, (e.g. Hall, 1988), i.e., $\sigma>1$; we maintain this assumption in the sequel, but we shall also briefly consider the commonly-used benchmark case of log-utility $(\sigma=1)$.

\section{Assumption 1: $\sigma>1$}

Our discount factor is given by:

$$
\Phi(\tau, t) \equiv \exp \{-\rho(\tau-t)\}(1+\delta(\tau-t))^{-\varphi / \delta}, \quad \rho, \varphi, \delta \geq 0
$$

which involves a mix of exponential and HD-based discounting: The former involves the standard discount rate, $\rho>0$; the latter is parameterised by (a) the strength of HD 
discounting, $\quad 0 \leq \varphi<1$, and (b) an index of present-bias, $\delta>0 .{ }^{5}$ Note that the exact functional form proposed here is one contribution of the present paper.

For the parameters shown, the discount factor (2) has the usual properties:

$$
\begin{aligned}
& \Phi(t, t)=1, \lim _{\tau \rightarrow \infty} \Phi(\tau, t)=0, \frac{d \Phi(\tau, t)}{d \varphi}<0, \frac{d \Phi(\tau, t)}{d \delta}>0 \\
& \lim _{\delta \rightarrow 0} \Phi(\tau, t)=\exp \{-(\rho+\varphi)(\tau-t)\}, \lim _{\delta \rightarrow \infty} \Phi(\tau, t)=\exp \{-\rho(\tau-t)\}
\end{aligned}
$$

The associated discount rate is:

$$
-\frac{\mathrm{d} \Phi(\tau, t)}{d \tau}=\rho+\frac{\varphi}{\Phi(\tau, t)}=\rho
$$

This discount rate begins high and asymptotically drops to the exponential discount rate $(\rho)$. As a result, the individual is more 'presently-biased', exhibiting higher impatience in any intertemporal dilemma the nearer it appears in the future. It is easy to check that a rise in $\delta$ decreases the distant-future discount rate in relation to the near-future one, i.e. makes the present-bias more pronounced.

The HD part of (2) is standard, see e.g. Al-Nowaihi and Dhami (2008). Our innovation is to combine this with an exponential part, and to restrict the configuration of parameters by Assumption 2. In the present framework, an exponential part is included both because of the need to have a positive discount rate asymptotically (see Proposition 1 and Corollary 1 below) and because in a growth context variables like consumption will be growing exponentially in the steady state; hence calculation of lifetime utility will necessarily involve integration of exponential and hyperbolic elements. In a recent contribution with a similar dynamic system as our own, Cabo, Martín-Herrán and Martínez-García (2016) have shown that nonexponential discounting facilitates the appearance of non-standard, non-exponential patterns of growth; but investigation of such possibilities is beyond our scope.

It should be noted that the issue of what exactly is the appropriate form of the discount factor in (2) is not settled (see, e.g., Frederick et al., 2002, footnote 13; O'Donoghue and Rabin, 2015; Findley and Caliendo, 2014). Following Loewenstein and Prelec (1992), al-Nowaihi and Dhami (2008) use a generalised hyperbola without any exponential portion. This however implies that the discount rate in the distant future is arbitrarily low, implying arbitrarily high patience asymptotically; there is no particular reason to think that this is the case. In general, Barro (1999) uses a hybrid exponential-hyperbolic formulation; for tractability, in parts of his analysis, he uses an exponential discount rate. As mentioned, the formulation of Cabo, Martín-Herrán and Martínez-García (2015) is more general but does not give rise to closed forms. Our formulation is a special case of Barro's (1999) general functional form that allows tractability (cf. Proposition 1).

\footnotetext{
${ }^{5}$ To use a metaphor, one knob $(\varphi)$ turns the water on-off while the other $(\delta)$ adjusts the temperature. We show that the effects of these parameters are quite similar; we refer to them (jointly and interchangeably) as the intensity of HD and/or present bias.
} 
In order to facilitate comparisons with the standard model, following Strulik (2015), we postulate a purely exponential discount rate $\psi$ that is equivalent in present-value terms to the mixed exponential-HD of (2); i.e., we define a $\psi>0$ such that:

$$
\int_{t}^{\infty} \exp \{-\psi(\tau-\mathrm{t})\} d \tau \equiv \int_{t}^{\infty} \Phi(\tau, t) d \tau
$$

As Appendix A shows in detail, under Assumption 2, the RHS of (3) can be integrated to yield:

$$
\psi=\frac{\delta^{\varphi / \delta} \rho^{1-\varphi / \delta}}{\Gamma(1-\varphi / \delta)}
$$

Obviously, $\lim _{\varphi \rightarrow 0} \psi=\rho$ : the purely exponential case emerges as a special case of our framework. Furthermore, as Appendix A shows, we also have:

$$
\frac{d \psi}{d \varphi}>0, \quad \frac{\mathrm{d} \psi}{\mathrm{d} \delta}>0
$$

$\psi$ increases with the intensity of HD and present bias. By defining a discount rate $(\psi)$ that does the same 'amount' of discounting in a present-value sense allows us to differentiate between the form and the 'amount' of discounting. We shall indicate as 'present value-equivalent' (superscripted 'PVEE') the propensity to consume $(\lambda)$ and the growth rate that would result from purely exponential discounting at rate $\psi$. Furthermore, for comparison, we shall also derive results under an exponential-only discounting scheme $(\varphi=0, \rho>0)$, indicated by superscript 'EXP'. Results under the PVEE and EXP schemes are to be contrasted with those under the hybrid scheme (superscripted 'HD'), which is our main focus.

The root of the time inconsistency problem is that, according to the scheme in (2), discounting between two future times $\tau^{\prime}>\tau>$ t depends not only on the difference $\tau^{\prime}-\tau$ (as in the exponential case) but also on the time of planning period (t) itself; to see this, write such discounting between these future dates evaluated now as:

$$
\bar{\Phi}\left(\tau^{\prime}, \tau, t\right) \equiv \frac{\Phi\left(\tau^{\prime}, t\right)}{\Phi(\tau, t)}=\exp \left\{-\rho\left(\tau^{\prime}-\tau\right)\right\} \frac{(1+\delta(\tau-t))^{-\varphi / \delta}}{\left(1+\delta\left(\tau^{\prime}-t\right)\right)^{-\varphi / \delta}}
$$

As a result, the perturbation argument underlying the standard Euler equation is not valid as the results depend on the horizon which we are considering. Developing the analyses of Strotz (1956) and Pollak (1968), Barro (1999) has presented a perturbation argument which is time-consistent as it depends on a 'policy' rule (consumption rule) and the resulting consumption dynamics that are both timeconsistent. We follow this procedure here.

In order for integration involving (2) to converge with sensible properties, we impose:

Assumption 2: $\varphi, \rho>0 ; \quad 0<\varphi / \delta<1 ; \quad \Delta / \exp \{1\}<\delta<\Delta$ for a $\Delta>1$ defined as: 


$$
\ln \Delta \equiv 1+\ln (\rho)-\frac{\Gamma^{\prime}\left(1-\frac{1}{\Delta}\right)}{\Gamma\left(1-\frac{1}{\Delta}\right)}
$$

where $\Gamma($.$) is the Gamma function.$

\section{Proposition 1:}

Under the parameter restrictions of Assumption 2, the discounting scheme (2) and (2') has a number of desirable properties:

- Tractability: it allows closed-formed solutions;

- Consistency: a rise in $\delta$ signifies both a rise in present-bias, and an increase in overall discounting in the sense that $\frac{\mathrm{d} \psi}{\mathrm{d} \delta}>0$;

- Generality: The model boils down to the purely exponential scheme when $\varphi=0$, which is therefore a special case of our framework.

Proof: Tractability is due to the fact that expressions involving (2) under Assumption 2 are integrable owing to $\varphi / \delta<1$; consistency: as mentioned, a rise in $\delta$ raises both the discount rate of the near future relative to that of the distant future, and increases the overall discount rate, $\psi$, see (3-3") below; generality: we show that the model's features boil down to those of the purely exponential scheme at key points below.

Notice that the restrictions in Assumption 2 resolve a tension: In this range of parameters, a rise in $\delta$ both increases present-bias but also discounts more heavily over the life time ( $\psi$ rises, see below). Outside it, a rise in $\delta$ increases the near-future discount rate in relation to the distant-future one (i.e., increases present-bias), but decreases overall discounting; which seems a contradiction. This ambiguity in the role of $\delta$ seems to have gone unnoticed in existing literature. Assumption 2 resolves it.

\section{2: A time-consistent procedure}

Following Barro (1999), the utility functional (1) may be linearised as:

$$
(1-\sigma) U_{t}=\varepsilon C_{t}^{1-\sigma}+\int_{t+\varepsilon}^{\infty} C_{\tau}^{1-\sigma} \Phi(\tau, t) d \tau
$$

This expresses intertemporal utility as two portions, a (linearised) flow of instantaneous utility for some for a sufficiently small period of time $\varepsilon>0$ ahead, and the standard utility functional thereafter (discounted at $\mathrm{t}$ ). The strategy is to consider a possible perturbation whereby the consumer changes consumption starting at $t$, and then uses the proceeds of that change to reset their consumption at $t+\varepsilon$ and beyond; this information is given by the consumer's budget constraint and some postulated but time-consistent consumption dynamics.

More formally, the perturbation should leave utility at time $\mathrm{t}$ unaltered, if the consumer is on an optimal path. Accordingly, the change in lifetime utility from a change in $\mathrm{C}_{\mathrm{t}}$ is: 


$$
\frac{d U_{t}}{d C_{t}}=\varepsilon C_{t}^{-\sigma}+\left\{\int_{t+\varepsilon}^{\infty} C_{\tau}^{-\sigma} \frac{d C_{\tau}}{d C_{t+\varepsilon}} \Phi(\tau, t) d \tau\right\} \frac{d C_{t+\varepsilon}}{d K_{t+\varepsilon}} \frac{d K_{t+\varepsilon}}{d C_{t}}=0
$$

This approach allows the entire stream of consumption, and not only consumption at a specific point in time as in the perturbation underlying the Euler equation, to be potentially affected after the initial change; this is key to delivering time consistency. The total effect on utility from a change in current consumption is made up of the sum of the direct effect on utility for as long as the initial change occurs $(\varepsilon)$ plus the effect on discounted utility from the change in consumption at $\tau \geq t+\varepsilon$. Furthermore, to gauge the change in future consumption, one needs an evolution formula for consumption from $t+\varepsilon$ onwards (the ratio inside the curly brackets), the effect of altered assets at $t+\varepsilon$ on consumption at $t+\varepsilon$ (a kind of propensity to consume out of assets, the first ratio outside the curly brackets), times the effect on assets at $t+\varepsilon$ from changed consumption at $\mathrm{t}$ (the second ratio). We consider these effects in turn.

The individual's budget constraint is expressed as:

$$
\frac{d K_{t}}{d t}=r_{t} K_{t}+W_{t}-C_{t}
$$

where $K_{t}$ are assets, $r_{t}$ is the rate of interest, and $W_{t}$ the labour income of the individual (all in real terms). The real interest rate need not be time-invariant; in fact, as it reflects the marginal productivity of capital, we shall take it as an exogenous driver of growth in what follows. Written as an approximation in terms of the small but finite period of time $\varepsilon$, this equation reads:

$$
K_{t+\varepsilon}=\left(1+\varepsilon r_{t}\right) K_{t}+\varepsilon W_{t}-\varepsilon C_{t}
$$

The labour income (W) will be linked to the production function, to be specified below. The main point here is, it is exogenous to the individual. Therefore,

$$
\frac{d K_{t+\varepsilon}}{d C_{t}}=-\varepsilon
$$

Evaluating the effect of higher assets at $\mathrm{t}+\varepsilon$ on consumption at that time and beyond $\left(d C_{t+\varepsilon} / d K_{t+\varepsilon}\right)$ is trickier. Following Barro (1999), we conjecture that there is a 'propensity to consume' out of current assets and life-time labour income, defined by:

$$
\lambda_{t} \equiv \frac{C_{t}}{K_{t}+\bar{W}_{t}}>0
$$

Formally, $\lambda_{\mathrm{t}}$ is defined as the consumption-intertemporal resources ratio; it will play a prominent role in what follows. It is allowed to be non-constant in dynamics, but will be the constant 'grand ratio' in the balanced growth path. Under purely exponential discounting, $\lambda_{t}$ reduces to the standard non-linear function of the interest rate and the exponential rate of time preference. Effectively, (6) gives the individual's hypothesised policy rule. Even though it is time-varying, the resulting outcome is time-consistent, as we explain below. ${ }^{6}$

\footnotetext{
${ }^{6}$ This is a good place to state more precisely a point of difference of our analysis with both Barro (1999) and Cabo et al. (2015). Our starting point (6), equivalently (6') is Barro's (1999) eq. (10) which is postulated under log-utility. We generalise that by considering more general preferences and
} 
(6) implies a consumption path equal to:

$$
C_{t}=\lambda_{t}\left(K_{t}+\bar{W}_{t}\right)
$$

Life-time labour earnings, $\bar{W}_{t}$, are defined as:

$$
\bar{W}_{t}=\int_{t}^{\infty} W_{\tau} \exp \left\{-\int_{t}^{\tau} r_{v} d v\right\} d \tau
$$

Thus, using (7), we obtain:

$$
\frac{d \bar{W}_{t}}{d t}=-W_{t}+r_{t} \bar{W}_{t}
$$

Using this and the budget constraint (5) into (6'), we get an expression for the dynamics of consumption:

$$
d C_{t} / d t=\left(r_{t}-\lambda_{t}\right) C_{t}+\left(d \lambda_{t} / d t\right) C_{t} / \lambda_{t}
$$

This equation replaces the Euler equation of consumption dynamics in the standard framework. The novelty of this setup in relation to standard theory is the existence of $\lambda_{t}$, as mentioned. Hence, consumption at any time $\tau \geq t+\varepsilon$ is given by:

$$
C_{\tau}=\exp \left\{\int_{t+\varepsilon}^{\tau}\left(r_{v}-\lambda_{v}+\dot{\lambda}_{v} / \lambda_{v}\right) d v\right\} C_{t+\varepsilon}
$$

with $\dot{\lambda}_{v} \equiv d \lambda_{v} / d v$.

Inserting the information from the budget constraint (5'), the policy rule (6') and consumption dynamics ( $\left.8^{\prime}\right)$, we are able to express the perturbation in (4) as:

$$
0=\varepsilon C_{t}^{-\sigma}-\varepsilon \lambda_{t+\varepsilon} C_{t+\varepsilon}^{-\sigma}\left\{\int_{t+\varepsilon}^{\infty} \exp \left[(1-\sigma) \Lambda_{\tau-\mathrm{t}-\varepsilon}\right] \Phi(\tau, t) d \tau\right\},
$$

where

$$
\Lambda_{\tau-\mathrm{t}-\varepsilon} \equiv \int_{t+\varepsilon}^{\tau}\left(r_{v}-\lambda_{v}+\dot{\lambda}_{v} / \lambda_{v}\right) d v
$$

Letting $\varepsilon \rightarrow 0$, we impose $\mathrm{C}_{\mathrm{t}+\varepsilon} \rightarrow \mathrm{C}_{\mathrm{t}}$, otherwise there would be discrete jumps in the path of consumption. Re-arranging (9), we get a value for the propensity to consume:

allowing for a variable $\lambda_{t}$ (constant under log-utility); differentiating (6'), we get (8). Note that under log-utility, (8) reduces to $\frac{d C_{t}}{d t}=\left(r_{t}-\lambda\right) C_{t}$, as $\lambda$ is constant; this is derived in Barro from (6' - his eq. 10). In considering more general preferences, however, Barro (1990, eq. 30) hypothesises an analogue to that in the manner of the Euler equation, which is $\frac{d C_{t}}{d t}=\frac{1}{\sigma}\left(r_{t}-\lambda_{t}\right) C_{t}$; and Cabo et al. (2015, eq. 6) use that. However plausible, this is not compatible with (6'), as can easily be checked. The main problem with this formulation is that it does not allow an interpretation of $\lambda$ as the consumptionintertemporal resources ratio (intuitively: the 'propensity to consume'); but, based on Barro's eq. (10), we do maintain this interpretation. Thus, our approach generalises that of Barro's Section 3, we therefore develop it explicitly. 


$$
\lambda_{t}=\frac{1}{\int_{t}^{\infty} \exp \left[(1-\sigma) \Lambda_{\tau-\mathrm{t}}\right] \Phi(\tau, t) d \tau} .
$$

Time-consistency has now been achieved: The propensity to consume $\lambda_{t}$ does not depend on $\tau$ (after integration), therefore it imposes the same rule on consumption at any given time irrespective of the timing of planning.

\section{3: Steady state}

Here, we characterise the steady state of equations (10) and (11). With constant $r$ and $\lambda$, the former becomes:

$$
\Lambda_{\tau-\mathrm{t}}=(\mathrm{r}-\lambda)(\tau-\mathrm{t})
$$

Introducing this into (11), we get:

$$
\lambda=\frac{1}{\int_{t}^{\infty} \exp \{\Omega(\tau-\mathrm{t})\}(1+\delta(\tau-t))^{-\varphi / \delta} d \tau},
$$

Where,

$$
\Omega \equiv-\rho+(1-\sigma)(r-\lambda)
$$

Integrating the denominator in (12) in a completely analogous manner to (3'), we get:

$$
\lambda=\frac{(-\Omega)^{1-\varphi / \delta}}{\Gamma(1-\varphi / \delta)} \delta^{\varphi / \delta}
$$

Equations (13) and (14) define a non-linear static system, depicted in Figure 1 in $(-\Omega$, $\lambda)$ space:

\section{Figure 1: The steady-state system}

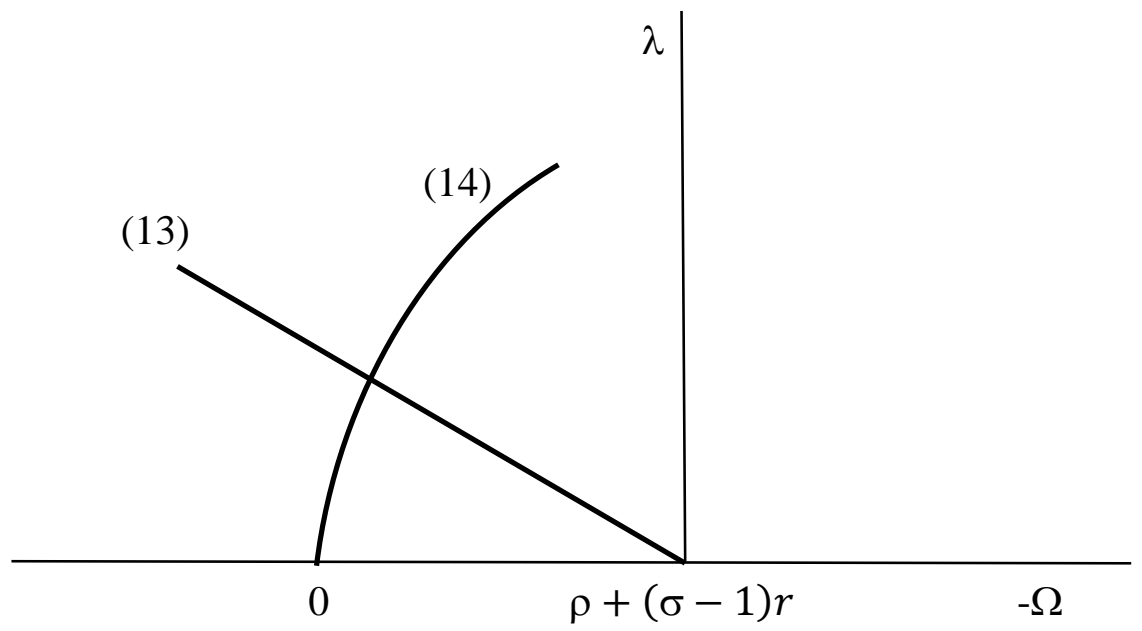


We obtain:

\section{Proposition 2:}

a) There exists a unique steady-state equilibrium $\left(\Omega^{H D}, \lambda^{H D}\right)$;

b) $0<\lambda^{H D}<r$;

c) $d \lambda^{H D} / d \varphi>0$;

d) $d \lambda^{H D} / d \delta>0$;

e) $d \lambda^{H D} / d \sigma>0$;

f) $d \lambda^{H D} / d \rho>0$;

g) $0<d \lambda^{H D} / d r<1$;

h) Under the EXP and PVEE schemes, $\lambda^{E X P}=\frac{\rho+(\sigma-1) r}{\sigma}$ and $\lambda^{P V E E}=\frac{\psi+(\sigma-1) r}{\sigma}$, resp.;

i) $\lambda^{E X P}<\lambda^{P V E E}<\lambda^{H D}$.

\section{Proof:}

a) A simple geometric argument based on Figure 1 establishes the existence and uniqueness of equilibrium: (14) is upward-sloping because of $0 \leq \varphi<1, \varphi / \delta<1$; while the slope of (13) is $-\frac{1}{(\sigma-1)}<0$ (from Assumption 1). As the loci are monotonic and with slopes of opposite signs, they do intersect once only in the positive quadrant;

b) See Appendix B;

c) By analogy with (3',), using Assumption 2;

d) By analogy with (3''), using Assumption 2;

e) We have from (13) that $\frac{\mathrm{d}\left(-\Omega^{\mathrm{HD}}\right)}{\mathrm{d} \sigma}=\mathrm{r}-\lambda^{\mathrm{HD}}-\sigma \frac{\mathrm{d} \lambda^{\mathrm{HD}}}{\mathrm{d} \sigma}$ and from (14) that $\frac{\mathrm{d} \lambda}{\mathrm{HD}}=\left(1-\frac{\varphi}{\delta}\right) \frac{\lambda^{\mathrm{HD}}}{-\Omega^{\mathrm{HD}}} \frac{\mathrm{d}\left(-\Omega^{\mathrm{HD}}\right)}{\mathrm{d} \sigma}$. Therefore, $\frac{\mathrm{d}\left(-\Omega^{\mathrm{HD}}\right)}{\mathrm{d} \sigma}=\frac{\mathrm{r}-\lambda^{\mathrm{HD}}}{1+\sigma\left(1-\frac{\varphi}{\delta}\right) \frac{{ }^{2} \mathrm{HD}}{-\Omega^{\mathrm{HD}}}}>0$, therefore $\frac{d \lambda^{H D}}{d \sigma}>0$ since by part (b) of the Proposition, $r-\lambda^{\mathrm{HD}}>0$;

f) A rise in $\rho$ shifts (13) right; therefore, the equilibrium 'slides' up (14), establishing the result;

g) A rise in $\mathrm{r}$ also shifts (13) right; by analogy with (e), the positive sign of the result follows. Furthermore, combining (13) and (14) we have:

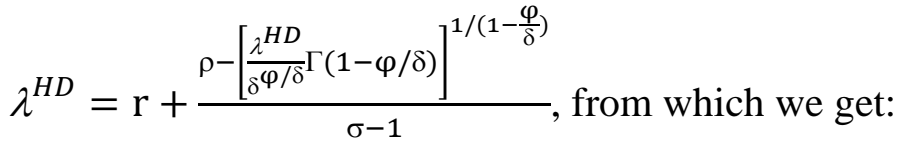

$\frac{d \lambda^{H D}}{d r}=1 /\left(1+\frac{1}{1-\frac{\varphi}{\delta}} \frac{(-\Omega)^{H D}}{\lambda^{H D}} \frac{1}{\sigma-1}\right)$. From (13), $\frac{(-\Omega)^{H D}}{\lambda^{H D}} \frac{1}{\sigma-1}=\frac{\rho}{(\sigma-1) \lambda^{H D}}+\frac{r}{\lambda^{H D}}-$ $1>0$. Therefore, the ' $<1$ ' part of the clause readily follows.

h) The PVEE and EXP special cases are defined by the following variants of (13) and (14) (setting $\varphi=0$ and using either $\psi$ or $\rho$ ):

$\Omega^{P V E E} \equiv-\rho+(1-\sigma)\left(\mathrm{r}-\lambda^{P V E E}\right)$

$\lambda^{P V E E}=-\Omega^{P V E E}+\psi-\rho$

and 


$$
\begin{aligned}
& \Omega^{E X P} \equiv-\rho+(1-\sigma)\left(\mathrm{r}-\lambda^{E X P}\right) . \\
& \lambda^{E X P}=-\Omega^{E X P}
\end{aligned}
$$

i) From $\left(13^{\prime} / 14^{\prime}\right)$ and $\left(13^{\prime}, / 14^{\prime \prime}\right)$, we see that

$$
\lambda^{\mathrm{EXP}}<\lambda^{\mathrm{PVEE}}
$$

follows trivially from $\psi>\rho$. We focus on $\lambda^{\mathrm{PVEE}_{<}} \lambda^{\mathrm{HD}}$ next. From (14) and (3') we have:

$\lambda^{H D}=\frac{\left(-\Omega^{H D}\right)^{1-\varphi / \delta}}{\Gamma(1-\varphi / \delta)} \delta^{\varphi / \delta}=\psi\left(\frac{-\Omega^{H D}}{\rho}\right)^{1-\varphi / \delta}>\psi$

The inequality follows from $\mathrm{r}>\lambda^{\mathrm{HD}}$ as is required for positive steady-state growth, therefore $-\Omega^{H D}>\rho$. On the other hand, from (13') and (14'), we get:

$$
\lambda^{P V E E}=\psi+(1-\sigma)\left(\mathrm{r}-\lambda^{P V E E}\right)<\psi
$$

This establishes that:

$$
\lambda^{\mathrm{PVEE}}<\lambda^{\mathrm{HD}}
$$

Apart from establishing the uniqueness and existence of steady-state equilibrium, Proposition 2 is key in several other respects. Part (b) implies the existence of a positive steady-state growth rate. Parts (c) and (d) jointly establish that a greater present-bias leads to more current (as opposed to future) consumption. Part (e) establishes that a rise in the intertemporal elasticity of substitution (decline in $\sigma$ ) decreases the current propensity to consume, an intuitive property (a rise in this elasticity implies that the individual can shift consumption towards the future by more if the incentives are right). Part (i), particularly inequality (16), is key, implying that the form of discounting matters and not just its 'quantity': The PVEE schemes and HD involve an equal amount of discounting in a present value sense, yet the latter implies a greater propensity to consume. By (8), this also yields a lower steady-state growth; we defer this discussion till later.

Furthermore, it is also straightforward to establish a further point related to the case of $\log$ utility $(\sigma=1)$; though not supported by evidence, this is a common benchmark in much theoretical work. We see that, if $\rho=0$ and $\sigma=1$ (against Assumption 1), (13) and (14) yield the trivial equilibrium $\lambda^{*}=\Omega^{*}=0$. To eliminate this possibility, we require $\rho>0$ :

\section{Corollary 1:}

In the case of $\log$ utility $(\sigma=1)$, the discount factor must contain a non-trivial exponential portion with a positive discount rate alongside the HD portion. 


\section{Short-run dynamics of $\lambda_{t}$}

\section{1: The system}

The dynamic equation is given by (11), using (10) and (2); it may expressed as the following system (letting $\varepsilon \rightarrow 0$ ):

$$
\begin{aligned}
& \lambda_{t}=\frac{1}{\int_{t}^{\infty} \exp \left[-\rho(\tau-\mathrm{t})+(1-\sigma) \Lambda_{\tau-\mathrm{t}}\right](1+\delta(\tau-\mathrm{t}))^{-\varphi / \delta} d \tau} . \\
& \Lambda_{\tau-\mathrm{t}} \equiv \int_{t}^{\tau}\left(r_{v}-\lambda_{v}+\dot{\lambda}_{v} / \lambda_{v}\right) d v .
\end{aligned}
$$

Linearising and expressing in terms of deviations from the steady state, we get the following dynamic equation:

$$
\dot{\lambda}_{t}=\lambda^{\mathrm{EXP}} \tilde{\lambda}_{t}+\frac{\lambda^{\mathrm{HD}}}{\sigma}(1-\sigma) \tilde{r}_{t}-\frac{\lambda^{\mathrm{HD}}}{\sigma}(1-\sigma) \gamma \tilde{\Lambda}_{\mathrm{t}}
$$

We use the following notational conventions:

- $\quad \lambda^{E X P} \equiv \frac{\rho-(1-\sigma) r}{\sigma}$, cf. (13'") and (14'");

- $\quad \lambda^{\mathrm{HD}}$ is the steady-state value of $\lambda_{\mathrm{t}}$ - the superscript differentiates it from $\lambda^{E X P}$;

- a tilde on the propensity to consume indicates a deviation from the steady state, i.e., $\tilde{\lambda}_{t} \equiv \lambda_{t}-\lambda$ and similarly with $\widetilde{\Lambda}_{t}$;

$-\quad \gamma \equiv \varphi \frac{\Gamma(1-\varphi / \delta)}{(-\Omega+\delta)^{1-\varphi / \delta} \delta^{\varphi / \delta}}$.

Appendix C reviews the properties of (20) in more detail.

From (10), we have in deviations form:

$$
\widetilde{\Lambda}_{\mathrm{t}} \equiv \int_{t}^{\infty}\left(\tilde{r}_{v}-\tilde{\lambda}_{v}+\dot{\lambda}_{v} / \lambda_{v}\right) d v
$$

implying:

$$
\dot{\tilde{\Lambda}}_{\mathrm{t}}=-\tilde{r}_{t}+\tilde{\lambda}_{t}-\dot{\lambda}_{t} / \lambda^{\mathrm{HD}}
$$

(19) and (21') can be consolidated as:

$$
\dot{\tilde{\Lambda}}_{\mathrm{t}}=-\frac{\tilde{r}_{t}}{\sigma}+\left(1-\frac{\lambda^{\mathrm{EXP}}}{\lambda^{\mathrm{HD}}}\right) \tilde{\lambda}_{t}+\frac{1-\sigma}{\sigma} \gamma \widetilde{\Lambda}_{\mathrm{t}}
$$

Equations (19) and (22) are a 2x2 first-order, linear differential system in $\tilde{\lambda}_{t}$ and $\widetilde{\Lambda}_{\mathrm{t}}$.

\section{2: The purely exponential special case}

It is instructive to review briefly the case of purely exponential discounting/no HD, shown by $\lambda^{\mathrm{HD}}=\lambda^{\mathrm{EXP}}$ and $\gamma=0$ in (22). In this case, the system collapses to: 


$$
\dot{\lambda}_{t}=\lambda^{E X P} \tilde{\lambda}_{t}+\frac{\lambda^{E X P}}{\sigma}(1-\sigma) \tilde{r}_{t}
$$

for which the solution is entirely forward-looking:

$$
\tilde{\lambda}_{t}=-\lambda^{E X P} \frac{(1-\sigma)}{\sigma} \int_{t}^{\infty} \exp \left\{-\lambda^{E X P}(v-t)\right\} \tilde{r}_{v} d v
$$

Thus, even in the absence of any present-bias, there will exist non-trivial, but entirely extraneous, dynamics of $\lambda_{t}$, providing it is purely exponential. There are two implications from this: The dynamics under purely exponential discounting is not different from the standard framework, where the exogenous driver also elicits purely extraneous dynamics. Secondly, the dynamics under PVEE will differ from the HD case although the 'amount' of discounting in the two cases is the same. Sub-Section 4.4 below shows that the dynamics in the HD case under an evolving external driver is not trivial and not purely extraneous.

In our framework, the dynamics of consumption is given by (8) and, evaluating at $\lambda_{t}=\lambda^{E X P}$, it may be re-written as:

$$
\frac{\dot{C}_{t}}{C_{t}}=\left(\tilde{r}_{t}-\tilde{\lambda}_{t}\right)+\frac{\left[\lambda^{E X P} \tilde{\lambda}_{t}+\frac{\lambda^{E X P}}{\sigma}(1-\sigma) \tilde{r}_{t}\right]}{\lambda^{E X P}}=\frac{\tilde{r}_{t}}{\sigma}
$$

This is standard. Thus, from the point of view of consumption dynamics as well, the standard theory is a special case of this framework under no HD.

\section{3: The general case: Properties of the system $(19,22)$ and diagrammatic exposition}

Appendix C has the full details. Under Assumption 1, the system's eigenvalues are real and of opposite signs, and are given by:

$$
2 q_{1,2}=\lambda^{E X P}+\frac{1-\sigma}{\sigma} \gamma \pm \sqrt{\left(\lambda^{\mathrm{EXP}}+\frac{1-\sigma}{\sigma} \gamma\right)^{2}-4 \frac{\lambda^{\mathrm{HD}}}{\sigma}(1-\sigma) \gamma}
$$

The eigenvalues have the following properties:

a) $0<q_{1}$;

b) $q_{2} \leq 0$ with equality when $\varphi=0$ (no present-bias).

The equilibrium is saddle-point stable; the full dynamics is described in Appendix C. The major point that emerges here is that the existence of the negative eigenvalue will generate non-trivial intrinsic dynamics, in sharp contrast to the counterpart case under purely exponential discounting. Below, we illustrate the dynamic adjustment; in Section 4, we outline the implications in the context of a simple 'AK' growth model. 


\section{Figure 2: Dynamics}

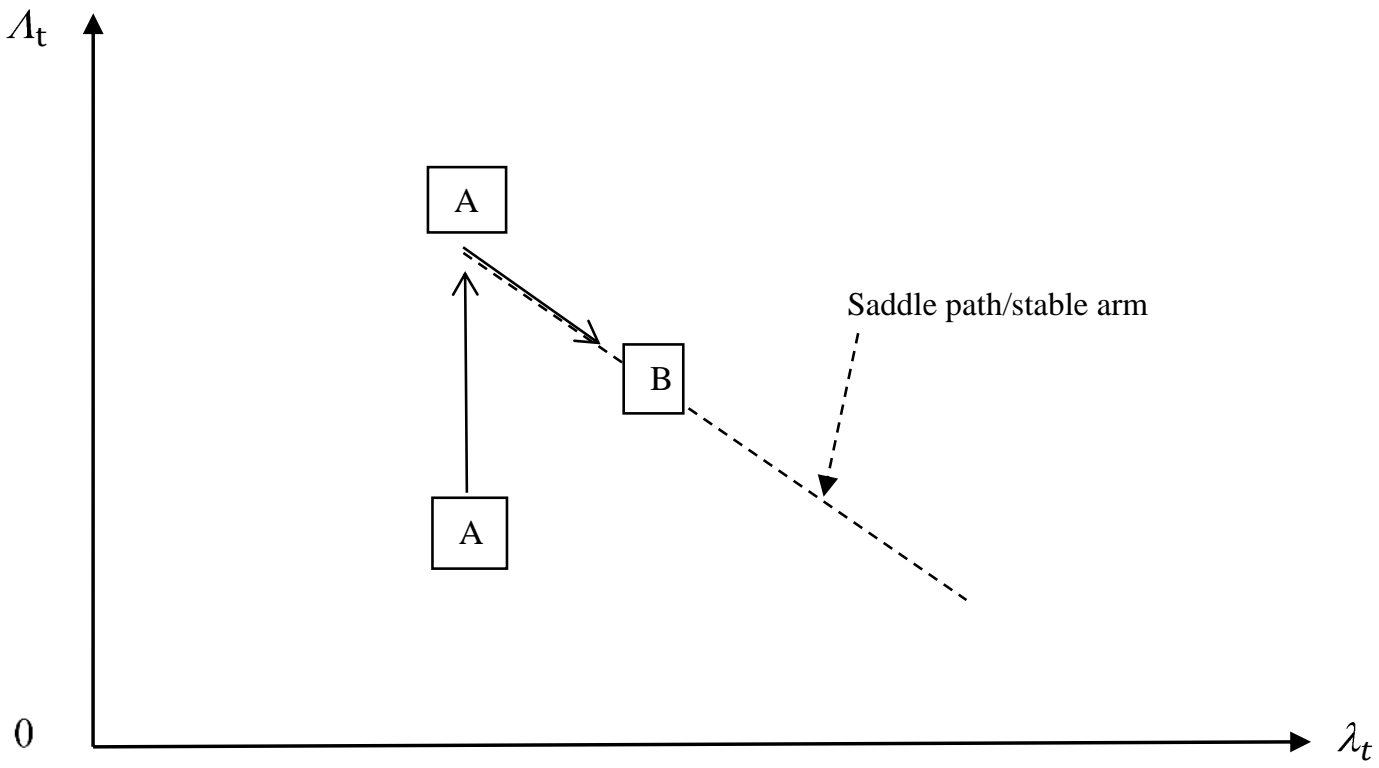

Figure 2 illustrates the saddle-point equilibrium implied by equations (19) and (22) in $\left(\lambda_{t}, \Lambda_{t}\right)$ space, under the textbook case of a constant external driver (here r). The stable arm is downward-sloping. For illustrative purposes, let A be the initial steady-state equilibrium and let the interest rate $r$ rise once-and-for-all. From Proposition $(2 \mathrm{~g})$ and (18), both $\lambda$ and $\Lambda$ rise in the steady-state; therefore the new equilibrium (B) will be northeast of A. Since the system is a saddle point, stability requires that there be a predetermined and a 'jump' variable (Turnovsky, 1995, Ch. 6; Buiter, 1982). We assume that $\lambda_{t}$ is a 'predetermined' variable, unable to jump and to cause any instantaneous jump in consumption; while $\Lambda_{t}$, as it is a forward sum, is able to change instantaneously. Note that this assumption may be underpinned by 'habits' in consumption (not modelled explicitly), which imply that individuals are reluctant to change consumption too much at any particular moment. Therefore, at the time of the exogenous change, there is an instantaneous jump up only of $\Lambda_{t}$; thereafter, $\lambda_{t}$ gradually increases and $\Lambda_{\mathrm{t}}$ decreases to correct the overshoot. The stable arm becomes flatter with a greater degree of present-bias; in that case, $\Lambda_{\mathrm{t}}$ jumps up by less and subsequently corrects by less; therefore $\left|\dot{\widetilde{\Lambda}}_{t}\right|$ decreases. This finding underpins some of the results below: To preamble, we shall see that the consumption-capital ratio rises, therefore a greater present bias leads to more consumption now and less saving and growth.

\section{4: The general case: analytical solution}

As Appendix D describes in full detail, the solution to system (19), (22) is given by:

$$
\tilde{\lambda}_{t}=c \mathrm{Q}_{2} \exp \left\{\mathrm{q}_{2} t\right\}+R_{1 t} \mathrm{Q}_{1} \exp \left\{\mathrm{q}_{1} t\right\}+R_{2 t} \mathrm{Q}_{2} \exp \left\{\mathrm{q}_{2} t\right\},
$$

and 


$$
\widetilde{\Lambda}_{t}=\operatorname{cexp}\left\{\mathrm{q}_{2} t\right\}+R_{1 t} \exp \left\{\mathrm{q}_{1} t\right\}+R_{2 t} \exp \left\{\mathrm{q}_{2} t\right\},
$$

where $\left[Q_{i} 1\right], i=1,2$ are the normalised eigenvectors corresponding to roots $q_{i}$, derived in Appendix C. The properties of $\mathrm{Q}_{1}$ and $\mathrm{Q}_{2}$ are defined in Appendix $\mathrm{D}$. Moreover, $R_{1,2 t}$ are defined as follows:

$$
\begin{aligned}
& R_{1 t} \equiv-\frac{\lambda(1-\sigma)+Q_{2}}{\sigma\left(Q_{1}-Q_{2}\right)} \int_{t}^{\infty} \exp \left\{-\mathrm{q}_{1} v\right\} \tilde{r}_{v} d v, \\
& R_{2 t} \equiv-\frac{Q_{1}+\lambda(1-\sigma)}{\sigma\left(Q_{1}-Q_{2}\right)} \int_{0}^{t} \exp \left\{-\mathrm{q}_{2} v\right\} \tilde{r}_{v} d v,
\end{aligned}
$$

c is a constant, to be determined as follows: As $\lambda_{t}$ does not change discretely, assuming that the exogenous driver (interest rate) is at a stationary equilibrium for $\mathrm{t}<\mathrm{T}$ and changes at $\mathrm{t}=\mathrm{T}$, we have the following expression for $\mathrm{c}$ :

$\tilde{\lambda}_{T}=c \mathrm{Q}_{2} \exp \left\{\mathrm{q}_{2} T\right\}-\exp \left\{\mathrm{q}_{1} T\right\} \mathrm{Q}_{1} \frac{\lambda^{\mathrm{HD}}(1-\sigma)+Q_{2}}{\sigma\left(Q_{1}-Q_{2}\right)} \int_{T}^{\infty} \exp \left\{-\mathrm{q}_{1} v\right\} \tilde{r}_{v} d v$

This is as there were no interest rate deviations before T. The value of $\tilde{\lambda}_{T}$ depends on the specific context; below, we turn to the AK model. After developing the essentials in sub-Sections 4.1-4, we continue the discussion of dynamics in sub-Section 4.5.

\section{Application: HD in a 'flexible AK' model}

\section{1: Setup:}

Consider the AK production function:

$$
Y_{t}=A_{t} K_{t},
$$

where $K_{t}$ is capital defined in a broad sense, and we assume that labour is inelastically supplied and equal to the time endowment of one. We let the marginal product of capital $A_{t}$ can vary over time; it is assumed proportional to the exogenous real interest rate, $\mathrm{r}_{\mathrm{t}}{ }^{7}$ To differentiate this setup from the standard AK model of a fixed productivity/interest rate, we call it a 'flexible AK' model:

$$
r_{t}=\alpha A_{t} .
$$

The economy-wide budget constraint is:

$$
\frac{d K_{t}}{d t}=Y_{t}-C_{t},
$$

\footnotetext{
${ }^{7}$ Implicitly, $0<\alpha<1$ is the share of privately owned physical capital in the broad concept of capital that is envisaged in the AK model; which is proportional to physical capital in the production function $(\mathrm{K})$.
} 
And the aggregate budget constraint is

$$
\frac{\dot{K}_{t}}{K_{t}}=\frac{r_{t}}{\alpha}-\frac{C_{t}}{K_{t}}
$$

\section{2: Steady-state results}

Growth is given by:

$$
\mathrm{g}^{\mathrm{HD}}=\mathrm{r}-\lambda^{\mathrm{HD}} \text {. }
$$

Considering Proposition 2, parts (b-h), we readily get:

Proposition 3: Determinants of the steady-state growth rate:
a) $d g^{H D} / d \varphi<0$;
b) $d g^{H D} / d \delta<0$;
c) $d g^{H D} / d \sigma<0$;
d) $d g^{H D} / d \rho<0$;
e) $0<d g^{H D} / d r<1$;
f) $g^{E X P}>g^{P V E E}<g^{H D}$

Proof: Consider (28) and Proposition 2.

Present bias increases the propensity to consume out of lifetime resources $(\lambda)$ and consequently decreases the growth rate. Importantly, the steady-state growth rate under HD is less than the purely exponential equivalent (PVEE) counterpart.

\section{Corollary 2:}

The form of discounting, as well as its amount, matters for the steady-state marginal propensity to consume and the growth rate.

Furthermore, we can derive the consumption-capital ratio: In the steady state, from (27') with (28) and dropping the HD subscript, we have:

$$
\frac{C}{K}=\frac{\mathrm{r}}{\alpha}-\mathrm{g}
$$

Considering Proposition 2, we unambiguously get the following results:

Proposition 4: Determinants of the steady-state consumption-capital ratio:
a) $d(C / K)^{H D} / d \varphi>0$;
b) $d(C / K)^{H D} / d \delta>0$;
c) $d(C / K)^{H D} / d \sigma>0$;
d) $d(C / K)^{H D} / d \rho>0$;
e) $(1-\alpha) / \alpha<d(C / K)^{H D} / d r<1 / \alpha$;
f) $(C / K)^{P V E E}<(C / K)^{H D}$. 
Proof: It follows from (27') with Proposition 2.

The consumption-capital $(\mathrm{C} / \mathrm{K})$ ratio is inversely indicative of the saving rate; this rises with technology (indicated by the real interest rate $r$ ) and falls with the discount rate; part (f) shows that saving is less under HD than with an equivalent (PVEE) amount of exponential discounting:

\section{Corollary 3:}

The saving rate is less under HD than with an equivalent (PVEE) amount of exponential discounting.

\section{3: The $r$-g relation}

In his recent, much talked about book, Picketty (2014) argues that the difference between the interest and the growth rates is crucial for the future of capitalism. Specifically, an $\mathrm{r}-\mathrm{g}>0$ will imply, the argument goes, that the share of capital in national income will perpetually rise; this is as this tends to get reproduced at the rate of the real interest rate (r) whereas real national income grows at rate g. Distributional considerations are outside the scope of this paper and we take no stance on Picketty's arguments here; but we note that they have already stirred a fair amount of debate, as exemplified e.g. in the Winter 2015 issue of the Journal of Economic Perspectives.

Our point here is that this model has interesting implications for the relation between these two variables. (28) readily gives:

$$
\mathrm{r}-\mathrm{g}=\lambda
$$

Therefore, our results can shed light on the 'r-g' wedge. We have:

\section{Corollary 4:}

a) The HD-related degree of present-bias raises the wedge between the real interest rate and the growth rate;

b) The HD-type of economy has a higher $\mathrm{r}-\mathrm{g}$ difference than an economy that discounts at an equivalent (in the PVEE sense) purely exponential rate.

As $\mathrm{r}$ is constant in the steady state, $\mathrm{r}-\mathrm{g}$ increases because of a decrease in $\mathrm{g}$. The implication is that, if Piketty's (2014) arguments are correct, the extent of present-bias will have important implications for the evolution of income distribution.

\section{4: Dynamics}

Re-writing the consumption dynamics (8) as:

$$
\frac{\dot{c}_{t}}{C_{t}}=r_{t}-\lambda_{t}+\dot{\lambda}_{t} / \lambda_{t}
$$


Next, combining (27') and (8''), we have:

$$
\frac{\dot{C}_{t}}{C_{t}}-\frac{\dot{K}_{t}}{K_{t}}=-\frac{1-\alpha}{\alpha} r_{t}+\frac{C_{t}}{K_{t}}-\lambda_{t}+\dot{\lambda}_{t} / \lambda_{t}
$$

Linearising and using $\dot{\widetilde{\Lambda}}_{\mathrm{t}}=-\tilde{r}_{t}+\tilde{\lambda}_{t}-\dot{\lambda}_{t} / \lambda$, we obtain:

$$
\frac{\dot{C}_{t}}{C_{t}}-\frac{\dot{K}_{t}}{K_{t}}=-\frac{1}{\alpha} \tilde{r}_{t}+\frac{C}{K}\left(1+\widetilde{\log C_{t}}-\widetilde{\log K}_{t}\right)-\dot{\widetilde{\Lambda}}_{\mathrm{t}}-\frac{(1-\alpha)}{\alpha} r-\lambda .
$$

This equation gives the dynamics of the consumption-capital ratio and depends crucially on the dynamics of $\widetilde{\Lambda}_{\mathrm{t}}$ derived above. ${ }^{8}$

Solving forward the deviations yields:

$$
\widetilde{\log C}_{t}-\widetilde{\log }_{t}=\int_{t}^{\infty} \exp \left\{-\frac{C}{K}(\tau-\mathrm{t})\right\}\left(\frac{1}{\alpha} \tilde{r}_{\tau}+\dot{\widetilde{\Lambda}}_{\tau}\right) d \tau
$$

The effect of $\dot{\widetilde{\Lambda}}_{\mathrm{t}}$ on the consumption-capital ratio is critical; it shows the channel by which present-bias affects the dynamics of the growth model. This necessitates another look at the dynamics of the marginal propensity to consume, undertaken in the next-sub-Section.

\section{5: Dynamics of $\tilde{\lambda}_{t}$ and $\widetilde{\Lambda}_{\mathrm{t}}$ (again)}

We now continue the discussion of the dynamics from where we left it at the end of Section 3. Since there is a close relationship between the real interest rate and productivity $\left(r_{t}=\alpha A_{t}\right)$, in what follows we shall take the interest rate as the exogenous driver of the dynamics: We consider exogenous changes in productivity (and the interest rate). We begin by definitising the constant $\mathrm{c}$ in the context of the initial condition (26a'). Following an unexpected, once-and-for-all rise in in steadystate interest rate at $\mathrm{t}=\mathrm{T}$, which will be reflected in a rise in steady-state from $\bar{\lambda}$ to $\overline{\bar{\lambda}}>\bar{\lambda},\left(26 \mathrm{a}^{\prime}\right)$ becomes:

$$
\mathrm{c}=\exp \left\{\left(\mathrm{q}_{1}-\mathrm{q}_{2}\right) T\right\} \frac{\mathrm{Q}_{1}}{\mathrm{Q}_{2}} \frac{\lambda(1-\sigma)+Q_{2}}{\sigma\left(Q_{1}-Q_{2}\right)} \int_{T}^{\infty} \exp \left\{-\mathrm{q}_{1} v\right\} \tilde{r}_{v} d v-\frac{\exp \left\{-\mathrm{q}_{2} T\right\}}{\mathrm{Q}_{2}} \Delta \lambda
$$

This is because, at time $\mathrm{t}=\mathrm{T}$, with $\lambda_{T}=\bar{\lambda}$ (no jump), the deviation is $\tilde{\lambda}_{T}=\lambda_{T}-\overline{\bar{\lambda}}=$ $\bar{\lambda}-\overline{\bar{\lambda}} \equiv-\Delta \lambda<0$, and the sign follows from the assumption that $\mathrm{r}$ jumps upwards. (It would have been the opposite if it had jumped downwards.)

Equations (26a, b) as well as (26a') depend on the deviations of the interest rate from its steady-state value. Therefore, one key result emerges immediately: When the interest rate jumps instantaneously to its steady-state value, there are no interest rate deviations at any time $\left(\tilde{r}_{v}=0, \forall \mathrm{t}\right)$, and the marginal propensity jumps freely to the new steady-state value. In this case, $c=0$ and the solution for $\tilde{\lambda}_{t}$ is,

\footnotetext{
${ }^{8}$ Since $\dot{\widetilde{\Lambda}}_{\mathrm{t}}=-\tilde{r}_{t}+\tilde{\lambda}_{t}-\dot{\lambda}_{t} / \lambda$, one may rely on either $\dot{\widetilde{\Lambda}}_{\mathrm{t}}$ or $\tilde{\lambda}_{t}-\dot{\lambda}_{t} / \lambda$ to solve; we choose the former.
} 


$$
\tilde{\lambda}_{t}=0, \forall \mathrm{t}
$$

i.e., in this case the model's dynamics degenerates: As in the standard AK model, the propensity to consume, and therefore the growth rate, jumps straight to the new steady-state values determined by the model's fundamentals (cf. Section 2 above).

But, under HD, this model deviates from the standard case if the external driver exhibits its own (extraneous) dynamics. For concreteness, let the interest rate rise after some time $\mathrm{T}$, but only gradually approach its steady state:

$$
\begin{array}{ll}
\mathrm{r}_{t}=\overline{\mathrm{r}}, & \mathrm{t}<\mathrm{T}, \\
\mathrm{r}_{t}=\overline{\mathrm{r}} \exp \{-\beta(t-T)\}+\overline{\overline{\mathrm{r}}}(1-\exp \{-\beta(t-T)\}), & \mathrm{t} \geq \mathrm{T}, \quad \overline{\overline{\mathrm{r}}}>\overline{\mathrm{r}}
\end{array}
$$

where $\beta>0$ is the speed of adjustment of $r_{t}$ from its former long-run equilibrium $(\bar{r})$ to the new one $(\overline{\overline{\mathrm{r}}})$. With $\beta \rightarrow \infty$, the driver exhibits an instantaneous jump to its new equilibrium (this is the textbook case of a once-and-for-all change). The case of $\overline{\overline{\mathrm{r}}}>\overline{\mathrm{r}}$ will be discussed in detail, while the symmetric opposite of a decrease in the interest rate will be discussed only briefly. Thus, the deviations are:

$$
\begin{array}{lr}
\tilde{r}_{t}=0, & \mathrm{t}<\mathrm{T}, \\
\tilde{r}_{t}=\mathrm{r}_{t}-\overline{\overline{\mathrm{r}}}=(\overline{\mathrm{r}}-\overline{\overline{\mathrm{r}}}) \exp \{-\beta(t-T)\} \leq 0, & \mathrm{t} \geq \mathrm{T}
\end{array}
$$

With these assumptions, from (26b), we get (see Appendix D for more details):

$$
\begin{gathered}
\widetilde{\Lambda}_{t}=-\exp \left\{\mathrm{q}_{2}(t-T)\right\} \frac{1}{\mathrm{Q}_{2}} \Delta \lambda+(\overline{\mathrm{r}}-\overline{\overline{\mathrm{r}}})\left[\exp \left\{\mathrm{q}_{2}(t-T)\right\}-\exp \{-\beta(t-T)\}\right] x \\
x\left[\frac{\lambda(1-\sigma)+Q_{2}}{\sigma\left(Q_{1}-Q_{2}\right)\left(\mathrm{q}_{1}+\beta\right)}-\frac{Q_{1}+\lambda(1-\sigma)}{\sigma\left(Q_{1}-Q_{2}\right)\left(\mathrm{q}_{2}+\beta\right)}\right]+ \\
+(\overline{\mathrm{r}}-\overline{\mathrm{r}}) \exp \left\{\mathrm{q}_{2}(t-T)\right\} \frac{\lambda(1-\sigma)+Q_{2}}{\sigma \mathrm{Q}_{2}\left(\mathrm{q}_{1}+\beta\right)}
\end{gathered}
$$

Therefore:

$$
\begin{gathered}
\dot{\widetilde{\Lambda}}_{\mathrm{t}}=-\mathrm{q}_{2} \exp \left\{\mathrm{q}_{2}(t-T)\right\} \frac{1}{\mathrm{Q}_{2}} \Delta \lambda+(\overline{\mathrm{r}}-\overline{\overline{\mathrm{r}}})\left[\mathrm{q}_{2} \exp \left\{\mathrm{q}_{2}(t-T)\right\}+\beta \exp \{-\beta(t-T)\}\right] x \\
x\left[\frac{\lambda(1-\sigma)+Q_{2}}{\sigma\left(Q_{1}-Q_{2}\right)\left(\mathrm{q}_{1}+\beta\right)}-\frac{Q_{1}+\lambda(1-\sigma)}{\sigma\left(Q_{1}-Q_{2}\right)\left(\mathrm{q}_{2}+\beta\right)}\right]+ \\
+\mathrm{q}_{2}(\overline{\mathrm{r}}-\overline{\mathrm{r}}) \exp \left\{\mathrm{q}_{2}(t-T)\right\} \frac{\lambda(1-\sigma)+Q_{2}}{\sigma \mathrm{Q}_{2}\left(\mathrm{q}_{1}+\beta\right)}
\end{gathered}
$$


Following a rise in the steady-state value of $\lambda$ due to a rise in the exogenous driver $r$, $\widetilde{\Lambda}_{t}$ jumps at $\mathrm{t}=\mathrm{T}$, thereafter asymptotically approaching zero.

As Appendix E shows, differentiating (32) with respect to the parameters of interest $(\varphi, \delta)$, evaluating at $\varphi \approx 0$, we obtain:

$$
\begin{aligned}
& \operatorname{sgn}\left\{\left.\frac{d \dot{\tilde{\Lambda}}_{\mathrm{t}}}{d \varphi}\right|_{\varphi=0}\right\}=\operatorname{sgn}(\overline{\overline{\mathrm{r}}}-\overline{\mathrm{r}}) \\
& \operatorname{sgn}\left\{\left.\frac{d \dot{\tilde{\Lambda}}_{\mathrm{t}}}{d \delta}\right|_{\varphi=0}\right\}=-\operatorname{sgn}(\overline{\overline{\mathrm{r}}}-\overline{\mathrm{r}})
\end{aligned}
$$

Aside from restricting attention to the vicinity of no present bias, results $\left(32^{\prime} \mathrm{a}, \mathrm{b}\right)$ come with two additional assumptions: that the rate of adjustment $(\beta)$ be high enough and that the time since the change (t-T) not be too high (see Appendix E). But it must be stressed that these restrictions are rather mild and not severely binding. Under these conditions, increasing present-bias will increase the value of $\dot{\tilde{\Lambda}}_{t}$ during the transition while the external driver is adjusting upwards. Things will be different when the driver adjusts downwards $(\overline{\overline{\mathrm{r}}}-\overline{\mathrm{r}}<0)$ : (32'a, b) imply that this will be lower with more HD-related short-termism. The next sub-Section will outline the wider implications of these results for the 'AK' growth model. But before, we summarise:

\section{Proposition 4: Dynamics of the propensity to consume $\left(\lambda_{t}\right)$ in the AK model:}

a) HD in general generates richer dynamics of the propensity to consume than under purely exponential discounting;

b) The dynamics degenerates in the special case of an infinite speed of adjustment of the external driver, $\beta \rightarrow \infty$, as e.g. in the textbook case of a onceand-for-all change in the exogenous fundamental (and without any habitsinduced inertia in the marginal propensity to consume, $\lambda_{t}$ );

c) There exists non-trivial but entirely extraneous dynamics in the case of sluggish adjustment (finite $\beta$ ) of the exogenous driver (interest rate) under a purely exponential discounting;

d) In the general case of less-than-infinite, but not too low, rate of adjustment $(\beta<\infty)$ and with present-bias, and in particular early on in the adjustment process ( $\mathrm{t}-\mathrm{T}>0$ not too high), if the exogenous interest rate increases (decreases) gradually, in the vicinity of no present-bias, a rise in HD-related present-bias increases (decreases) $\dot{\widetilde{\Lambda}}_{\mathrm{t}}$;

e) Under (d) above, the presence of endogenous dynamics is due to the form rather than the amount of discounting: The dynamics under the HD scheme is qualitatively different than under the PVEE scheme.

Proof: See the preceding discussion.

\section{6: Consumption and growth dynamics}


Proposition (4d) applied to (30) leads to a major result: In the case of a rise (fall) in the exogenous interest rate, a rise in present bias implies that the consumption-capital ratio is higher (lower) than otherwise, as $\dot{\widetilde{\Lambda}}_{\tau}$ rises (falls); intuitively, consumption is more front-loaded in anticipation of the future rise (fall) in capital and available resources. Hence, $\frac{C_{t}}{K_{t}}$ is more intensely pro-cyclical (its variance rises) and 'leading' (it rises or falls ahead of the rise in productivity/interest rate). Note that this effect is induced by HD-related present-bias only, and is unrelated to the exponential part of discounting, no matter how intense. These effects are of course derived under the presumption that productivity (as reflected in the real interest rate) is the only exogenous driver. A voluminous business cycles research (particularly 'Real Business Cycles' models, e.g. King and Rebelo, 1999) has argued that this is indeed the main driver. Future research following this paper can establish whether the results derived here for illustration purposes, under the interest rate as the sole driver, hold under other external drivers. We can partially summarise as follows:

\section{Proposition 5: Business cycles effects of $\mathrm{HD}$ :}

a) When the exogenous interest rate is rising (falling), a rise in the degree of HDrelated present-bias will generate a rise (fall) in the consumption-capital ratio and a reduction (rise) in the capital growth rate during the transition process.

b) Under the assumption that the real interest rate (reflecting productivity) is the sole driver of business cycles:

$\circ$ More intense HD results in the consumption-capital ratio being more intensely procyclical with a higher variance;

- These effects are due only to the form of discounting; thus, an economy with more intense HD will have a more procyclical consumption-capital ratio than an economy with an equivalent (in the PVEE sense) amount of discounting.

Proof: Consider the preceding discussion.

Note that since $r_{t}=\alpha A_{t}$, these business cycle-related results carry over to the consumption-output ratio; in deviations, this ratio is given by the following modification to (30):

$$
\begin{array}{r}
\widetilde{\log C}_{t}-\widetilde{\log Y}_{t}=\widetilde{\log C}_{t}-\widetilde{\log K}_{t}-\tilde{r}_{t}= \\
=\int_{t}^{\infty} \exp \left\{-\frac{c}{K}(\tau-\mathrm{t})\right\}\left(\frac{1}{\alpha} \tilde{r}_{\tau}+\dot{\widetilde{\Lambda}}_{\tau}\right) d \tau-\tilde{r}_{t}
\end{array}
$$

As the interest rate is exogenous, none of the above effects of present-bias on the ratio via $\dot{\widetilde{\Lambda}}_{\tau}$ is to be altered.

The increased procyclicality of consumption relative to output following an intensification of HD-related present-bias is interesting from yet another perspective. Standard models have some difficulty accounting for the fact that consumption is very procyclical and almost tracks income (the 'excess sensitivity result' or 'consumptionoutput puzzle'). For instance, King and Rebelo (1999, Table 1) shows a standard deviation of consumption relative to that of output of 0.74 in the data, whereas their 
baseline RBC model predicts 0.44 (Table 3). Our results confirm the finding first suggested by Laibson (1997) that the presence of present-bias and non-exponential discounting may be able to account for the discrepancy. Intuitively, this is because in this case consumption is driven less by permanent-income and more by current income considerations. We have:

\section{Corollary 5:}

HD-related present-bias gives rise to a more procyclical and variable consumptionoutput ratio, potentially resolving a long-standing macroeconomics puzzle.

This leads us on to the implications for the growth rate of capital, to be gauged from the above in relation to the aggregate budget constraint (27). As the rate of capital accumulation equals productivity (proportional to the interest rate) minus the consumption-capital ratio, the rise (fall) induced by present-bias to the $\frac{C_{t}}{K_{t}}$ ratio when the interest rate rises (falls) implies that the rate of capital accumulation correspondingly falls (rises). (Under plausible parameter values $\frac{r_{t}}{\alpha}-\frac{C_{t}}{K_{t}}>0$ always.) Thus, the enhanced pro-cyclicality of the consumption-capital ratio due to present bias leads to a reduced pro-cyclicality and a reduced variance of the rate of capital accumulation.

From here, it is a small step to show that the level of the capital stock will in fact be lower under a more intense HD. Writing that level as,

$$
\log K_{t}=\log K_{0}+\int_{0}^{t} \frac{\dot{K}_{\tau}}{K_{\tau}} d \tau
$$

we can express log-capital as the sum of an exogenous endowment at the beginning of time plus the growth rate ever since. If we approximate, ${ }^{9}$

$$
\int_{0}^{t} \frac{\dot{K}_{\tau}}{K_{\tau}} d \tau \approx t E\left(\frac{\dot{K}_{\tau}}{K_{\tau}}\right)+\frac{t^{2}}{2} \operatorname{Var}\left(\frac{\dot{K}_{\tau}}{K_{\tau}}\right)
$$

we immediately see that the reduction in the variance of capital accumulation due to more intense HD will in fact reduce that overall amount of capital accumulation and the level of capital stock. Intuitively, the percentage decline in $\mathrm{I} / \mathrm{K}$ during a boom, when capital is high, minus the (equiproportional) rise in the same ratio during a trough, when capital is low, leaves a negative net effect on capital accumulation. This is a levels effect additional to the steady-state effect on the growth rate discussed in sub-Section 4.2 above (which would show up in the expectations term): Economies with more intense HD are less capitalised and poorer in relation to economies with an equivalent (PVEE) amount of purely exponential discounting. This is yet another important implication of HD, with relevance to cross-country income distribution.

The last result merits a highlight in a separate Corollary:

\section{Corollary 6:}

\footnotetext{
${ }^{9}$ This equation will be accurate in the case of log-normality, otherwise an approximation.
} 
a) Present-biased economies, in the sense of HD discounting, differ in their degree of capitalisation even if their steady-state growth rates are identical.

b) An HD-related present-biased economy will be less capitalised in the steady state than an exponentially discounting economy that discounts at a present value-equivalent (PVEE) rate $(\psi)$.

c) The form of discounting, particularly the presence of HD, may account for parts of the capital and income disparities between richer and poorer countries.

These results have implications for development accounting and cross-country income inequality in so far as in any historical sample, the level of development is likely to be a mixture of steady-state growth and transitional dynamics.

Finally, we come to the question of 'observational equivalence' between hyperbolic and exponential discounting, which comes in two forms, 'weak' - whether $a$ (any) purely exponential discount rate exists that replicates the results under hyberbolic discounting, and 'strong' - whether the PVEE rate replicates these results. Previous literature has taken a mixed stance, no doubt attributable to the different setups employed. E.g., Barro (1999) shows that strong equivalence is preserved for log utility in a neoclassical growth model and a sophisticated bias; Strulik (2015) shows the same for the AK models under naïve bias; but that does not hold true in general as shown by Farzin and Wedner (2014) under isoelastic utility for a neoclassical model and naïve bias. Under a fairly general setup with and AK model, Cabo, Martin-Herran and Martinez-Garcia (2015) find weak equivalence for both naïve and sophisticated bias and strong equivalence under a sophisticated bias. Drawing on all our results, we have found that present-biased economies have different both steady-state properties and cyclical properties from purely exponentially-discounting economies, even from those discounting with a PVEE discount rate. ${ }^{10}$ The behaviour of HD-type economies cannot be replicated by 'exponential' economies, no matter what the exponential discount rate is.

\section{Corollary 7:}

There is no 'observational equivalence' between HD and purely exponentiallydiscounting economies.

\section{Conclusions}

This paper integrates hyperbolic discounting (HD) into the theory of growth in a continuous-time, deterministic framework when no commitment is assumed. It uses the methods of Barro (1999), who in turn builds on earlier work by Strotz (1956) and Pollak (1968), for dealing with the time-inconsistency problem that is inherent in any non-exponential discounting. The way time-inconsistency is dealt with is by foregoing entirely the Euler equation; instead, a dynamic equation is derived of the propensity to consume (consumption-to-lifetime resources ratio). Following Barro

\footnotetext{
${ }^{10}$ It cannot be precluded that some exponential discount rate exists that replicates the steady-state results obtained here. But the results related to transitional dynamics are qualitatively different arguing against equivalence of any kind.
} 
(1999), the paper allows for HD discounting in addition to exponential discounting. Our contribution may be partly summarised as extending the results of Barro, Strulik (2015) and Cabo, Martín-Herrán and Martínez-García (2015) by allowing for isoelastic utility, deriving sharper results on the time-consistent procedure under a 'sophisticated' present bias and expressly linking those to growth (the AK model). We also derive the implications for growth dynamics. All our results are derived analytically. In all these respects, we go beyond existing literature. In addition, this setup provides the foundations for an extension to the flexible labour case, thus more firmly embedding it into the theory of growth.

The discounting scheme is flexible enough yet lends itself to closed-form solutions and is an innovation of this paper. It is an exponential-HD hybrid characterised by an exponential discount rate $(\rho)$, an intensity of HD parameter $(\varphi)$ and the degree of present-bias $(\delta)$. It includes the standard pure exponential as the special case of $\varphi=0$. To sharpen the questions we ask, we postulate a 'present value-equivalent' (PVEE) purely exponential discount rate that does the same overall amount of discounting as our scheme. In this way, we are able to distinguish between the effects of present-bias per se from the amount of discounting. We derive a wide range of results related to both the hypothesised propensity to consume $(\lambda)$ per se, but also related to growth and the dynamics of business cycles. Our results consistently point to one conclusion, namely that the form and not only the amount of discounting matters: There is no observational equivalence between HD and exponential discounting as is sometimes argued.

We find that the existence of HD-related present-bias matters for the long run properties of the system: The degree of present-bias increases the marginal propensity to consume out of lifetime resources $(\lambda)$, decreases the growth and saving rates and increases the difference between the real interest rate and the growth rate $(\mathrm{r}-\mathrm{g})$, a quantity argued by Piketty (2014) to predict the long-term evolution of income distribution. Moreover, these effects are more pronounced than under PVEE.

Our setup also generates potentially non-standard dynamics. The dynamics may be decomposed into the dynamics of the propensity to consume and the dynamics of the full (otherwise standard AK) growth model. This framework implies endogenous dynamics of the propensity to consume in the case of a finite rate of adjustment of the external driver (for illustration here: the interest rate, reflecting productivity). The dynamics degenerates in the special case of an infinite speed of adjustment of the external driver, as e.g. in the textbook case of a once-and-for-all change in the exogenous fundamental, and becomes completely exogenous in the case of purely exponential discounting. Outside these special cases, the dynamics of the propensity to consume becomes richer with the existence of HD-related present-bias.

The implications for growth are illustrated by grafting the framework just discussed onto the AK growth model. Aside from the special cases mentioned above, the consumption-capital ratio and the growth rate will be affected by a rise in HD-related present-bias during transition as well as in the steady state. The results for growth dynamics are derived in the vicinity of no HD or present-bias $(\varphi \approx 0)$ and under some additional, rather mild assumptions (high enough $\beta$ and low enough t-T). In the case of a rise (fall) in the interest rate, a rise in the present-bias will generate a rise (fall) in the consumption-capital ratio and a reduction (rise) in the capital growth rate during 
the transition process. Thus, when the exogenous driver is productivity (captured by the real interest rate) as has been assumed here, partly guided by much business cycles research and partly for illustration purposes, the consumption-capital and consumption-output ratios become more volatile and procyclical, as consumption is driven more by current than permanent income. We argue that this result can potentially resolve a long-standing 'consumption-output puzzle', namely the difficulty by standard models to account for the high volatility and 'excess sensitivity' of consumption; by raising the predicted volatility, our model shows that present-bias can bring the predicted closer to the data. Furthermore, present-biased economies have a lower (average) steady-state level of capital; this is in addition to the steadystate growth rate effect of the present-bias. This result, as well as the results on steady-state growth, will have implications for cross-country income inequality. A final remark is that the transitional dynamics and business cycles-related results are not present at all under purely exponential discounting; therefore, an economy with an equivalent amount (in the PVEE sense) of purely exponential discounting will not exhibit these features at all.

In sum, we find that present-bias in discounting under no commitment has important implications for both long-term growth and business cycle-related dynamics, saving, development and for intra- or inter-country income inequality. These results should be of acute interest to both theorists and policy-makers.

\section{References:}

Ainslie, G.W. (1992): Picoeconomics, Cambridge, UK: Cambridge UP.

al-Nowaihi, A. and S. Dhami (2008): A note on the Loewenstein-Prelec theory of intertemporal choice, Mathematical Social Sciences, 52, 1 (July), pps. 99-108.

Angeletos, G.-M., D. Laibson, A. Repetto, J. Tobacman and S. Weinberg (2001): The Hyperbolic Consumption Model: Calibration, Simulation, and Empirical Evaluation, The Journal of Economic Perspectives, 15, 3 (Summer), pps. 47-68.

Barro, R.J. (1999): Ramsey meets Laibson in the neoclassical growth model, Quarterly Journal of Economics, 114, 4 (November), pps. 1125-52.

Beavis, B. and I. Dobbs (1990): Optimization and stability theory for economic analysis, Cambridge, UK: Cambridge University Press.

Buiter, W.H. (1982): Predetermined and non-predetermined variables in rational expectations models, Economics Letters, 10 (1-2), pps. 49-54.

Cabo, F., G. Martín-Herrán and M.P. Martínez-García (2015): Non-constant discounting and Ak-type growth models, Economics Letters, 131, pps. 54-8.

Cabo, F., G. Martín-Herrán and M.P. Martínez-García (2016): Unbounded growth in the Neoclassical growth model with non-constant discounting, Mathematical Social Sciences, 84, pps. 93-104.

Caliendo, F.N. and D. Aadland (2007): Short-term planning and the life-cycle consumption puzzle, Journal of Economic Dynamics and Control, 31, 4 (April), pps. 1392-1415.

DellaVigna, S. and U. Malmendier (2006): Paying not to go to the gym, American Economic Review, 96 (3), pps. 694-719

DellaVigna, S. (2009): Psychology and Economics: Evidence from the Field, Journal of Economic Literature, 47, 2, pps. 315-372.

Drouhin, N. (2009): Hyperbolic discounting may be time consistent, Economics Bulletin, 29, pps. 2549-2555.

Dyke, P. (2014): An Introduction to Laplace Transforms and Fourier Series, Springer. 
Farzin, Y.H. and R. Wendner (2014): The Time Path of the Saving Rate: Hyperbolic Discounting and Short-Term Planning, FEEM WP No. 063.2014; available at SSRN: http://ssrn.com/abstract=2463593 or http://dx.doi.org/10.2139/ssrn. 2463593

Findley, T.S. and F.N. Caliendo (2014): Interacting mechanisms of time inconsistency, Journal of Economic Psychology, 41, pps. 68-76.

Frederick, S., G. Loewenstein, T. and O'Donoghue (2002): Time Discounting and Time Preference: A Critical Review, Journal of Economic Literature 40, 2 (June), pps. 351401.

Hall, R.E. (1988): Intertemporal Substitution in Consumption, Journal of Political Economy, 96, 2 (April), pps. 339-57.

Harris, C. and D. Laibson (2001): Dynamic Choices of Hyperbolic Consumers, Econometrica, 69, 4 (July), pps. 935-57.

King, R.G. and S.T. Rebelo (1999): Resuscitating real business cycles, in: J.B. Taylor and M. Woodford (ed.), Handbook of Macroeconomics, vol. 1, Ch. 14, pps. 927-1007 Elsevier.

Krusell, P.B. and A.A. Smith, Jr. (2003): Consumption--Savings Decisions with QuasiGeometric Discounting, Econometrica, 71, 1 (January), pps. 365-375.

Laibson, D. (1997): Golden Eggs and Hyperbolic Discounting, Quarterly Journal of Economics, 112, pps. 443-77.

(2015): Why Don't Present-Biased Agents Make Commitments?, American Economic Review: Papers \& Proceedings, 105, 5 (May), pps. 267-72

Loewenstein, G. and D. Prelec (1992): Anomalies in Intertemporal Choice: Evidence and an Interpretation, Quarterly Journal of Economics, CVII, pps. 573-98.

O'Donoghue, T. and M. Rabin (2015): Present Bias: Lessons Learned and To Be Learned, American Economic Review: Papers \& Proceedings, 105, 5 (May), pps. 273-9

Phelps, E. S. and R. A. Pollak (1968) :On Second-best National Saving and GameEquilibrium Growth," Review of Economics Studies, 35, pps. 185-99.

Picketty, T. (2014): Capital in the Twenty-First Century, Boston, MA: Belknap (Harvard) Press

(2015): Putting Distribution Back at the Center of Economics: Reflections on Capital in the Twenty-First Century, Journal of Economic Perspectives, 29, 1 (Winter), pps. 67-88.

Pollak, R.A. (1968): Consistent Planning, Review of Economic Studies, 23, 3 (April), pps. 201-8.

Spear, Stephen E., (1991): Growth, externalities, and sunspots, Journal of Economic Theory, 54, 1 (June), pps. 215-23.

Sprenger, C. (2015): Judging Experimental Evidence on Dynamic Inconsistency, American Economic Review: Papers and Proceedings, 105, 5 (May), pps. 280-5

Strotz, R.H. (1956): Myopia and Inconsistency in dynamic Utility Maximization, Review of Economic Studies, 23 (3), pps. 165-80.

Strulik, H. (2015): Hyperbolic discounting and endogenous growth, Economics Letters, 126, pps. $131-4$.

Thaler, R. H. (1981): Some Empirical Evidence on Dynamic Inconsistency, Economic Letters 8 (3), pps. 201-207.

Tournemaine, F. and C. Tsoukis (2015): The growth-distribution nexus in a mixed regime of education with a status motive: On the macroeconomics of the welfare state, Economic Modelling, 47, June, pps. 235-43.

Tsoukis, C. (2014): Status, hyperbolic discounting, growth, and distribution, mimeo, July.

Turnovsky, S. (1995): Methods of Macroeconomic Dynamics, Cambridge, MA: MIT Press.

Zou, Z., S. Chen, W. Lei (2014): Finite horizon consumption and portfolio decisions with stochastic hyperbolic discounting, Journal of Mathematical Economics, 52, May, pps. 70-80. 